\title{
Multiple-color GaAs/AlGaAs superlattice infrared photodetector controlled by the polarity and magnitude of the bias voltage
}

\author{
M. C. Hsu and C.H. Kaun \\ Department of Electrical Engineering, National Taiwan University, Taipei, Taiwan \\ S.Y. Wang and C.P. Lee \\ Department of Electronics Engineering, National Chiao Tung University, Hsinchu, Taiwan
}

\begin{abstract}
We present a voltage-controlled multi-color infrared photodetector consisting of two stacks of GaAs/AlGaAs superlattices with main absorption peaks at $10.7 \mu \mathrm{m}$ and $6.7 \mu \mathrm{m}$ respectively. The switching in response wavelengths is achieved by using the opposite polarity dependence of responsivity in each stack. Compared with traditional voltage-controlled designs, the detector achieves better switching in response colors and lower operating bias voltage.
\end{abstract}

\section{Introduction}

Multicolor infrared detectors have several advantages over conventional ones. For example, making use of the different values of the emissivity among objects at specific wavelengths, we can use multicolor detectors to identify objects. Since the first observation of the intersubband transition [1], quantum well infrared detectors have been demonstrated with high detectivity and uniformity for different detection ranges [2]. Because of the flexibility in tailoring the subband levels of quantum wells, which determine the detection range, multicolor detectors based on intersubband transitions are easier to implement than other type of infrared detectors.

Many voltage-tunable multicolor intersubband infrared detectors have been proposed [3-7]. Some of them used the stark effect in coupled quantum wells [3] or asymmetric quantum wells [4]. However, the reported tuning ranges are limited and unable to cover a wide detection range. Another simple approach, which is more suitable for the detection covering a wide spectral range, is to stack several MQW layers, each with a specific detection range [5-7]. In the conventional designs of this approach, typically a two-stack detector can achieve only two-color sensing. The bias voltage level required to turn on a long-wavelength stack is usually high [5]. The high voltage requirement for the switching in colors will be more serious for multicolor detectors, which need more stacks to achieve more colors. In addition, in most cases the response from an individual stack can not be well separated from that of other stacks $[5,7]$.

In this study, we propose the use of a superlattice infrared photodetector (SLIP) for the stacked component in a two-stack infrared photodetector to achieve better switching in colors and lower operating bias voltage. The proposed SLIP consists of a supérlattice active layer followed by a thick blocking layer. According to our pervious study [8], the introduced blocking layer will cause the photocurrent to have a preferred direction and be nearly prohibited in the reverse direction. The polarity dependence of the photocurrent provides a mechanism to control the response of a stacked SLIP to be on or off. Therefore, the response of different stacks can be well separated by controlling the bias polarity. The bias voltage required to turn on the long-wavelength stack can also be reduced. In addition, the spectral response of such a SLIP is also tunable by changing the bias voltage dropped on its blocking layer. At a low bias voltage level, only those high-energy photoexcited carriers can pass through the energy barrier of the blocking layer. The resulting peak wavelength is associated with the Brillouin zone (BZ) center transition, from the first miniband minimum to the second miniband maximum. At a higher bias voltage level, the energy barrier becomes nearly transparent for all photoexcited carriers. Due to the larger oscillator strength, the zone edge transition, from first miniband maximum to second miniband minimum, becomes dominant. By stacking two such SLIPs in a back-to-back configuration, we demonstrate a multicolor detector that can be controlled by the polarity and magnitude of the bias voltage.

\section{Experiments}

The sample was grown by molecular beam epitaxy on a semi-insulating substrate. A $5000-\AA \mathrm{GaAs}$ layer with $\mathrm{Si}$ doped to $1 \times 10^{18} / \mathrm{cm}^{3}$ was first grown on the substrate as the bottom contact layer. And then the SL layer of the bottom SLIP designed for long-wavelength detection was grown followed by a $1500-\AA$ undoped $\mathrm{Al}_{0.2} \mathrm{Ga}_{0.8} \mathrm{As}$ blocking layer. After the bottom SLIP, a $5000-\AA \mathrm{n}^{+}(1 \times$ $10^{18} / \mathrm{cm}^{3}$ ) GaAs layer was grown to connect the bottom SLIP and top SLIP. The blocking layer of the top SLIP, 1000 - $\AA$ undoped $\mathrm{Al}_{0.34} \mathrm{Ga}_{0.66} \mathrm{As}$, was then grown and followed by the SL layer of the top SLIP designed for a shorter wavelength range. Finally a $3000-\AA \mathrm{n}^{+} \mathrm{GaAs}$ layer doped to $1 \times 10^{18} \mathrm{~cm}^{-3}$ was grown for the top 


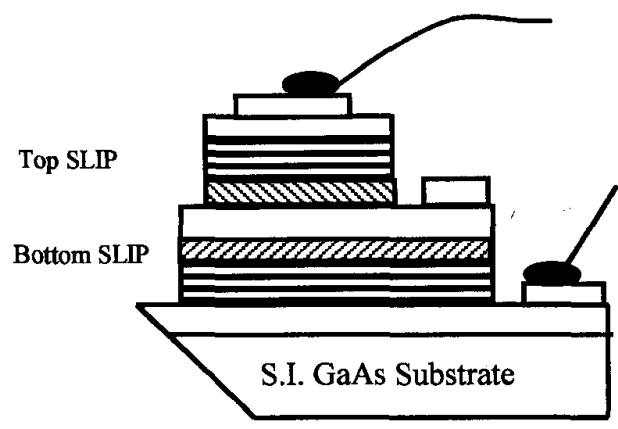

Fig. 1. Schematic structure of the two-stack SLIP. In the voltage-controlled operation, the bias is applied between top and bottom contacts.

contact. The top SL layer consists of 10 periods of $45-\AA$ undoped $\mathrm{Al}_{0.34} \mathrm{Ga}_{0.66} \mathrm{As}$ and $40-\AA \AA \AA \mathrm{GaAs}\left(\mathrm{n}=1 \times 10^{18} \mathrm{~cm}^{-3}\right)$. The bottom SL layer consists of 10 periods $68-\AA$ GaAs $\left(\mathrm{n}=1 \times 10^{18} \mathrm{~cm}^{-3}\right)$ and $35-\AA$ undoped $\mathrm{Al}_{0.27} \mathrm{Ga}_{0.73} \mathrm{As}$. The SL structures were designed according to the transfer matrix technique with taking the non-parabolicity of conduction band into account [9]. Although the sample was designed to be a voltage-controlled device, which requires only two contacts, we processed our sample into a three-terminal device as shown in Fig. 1 to allow the characterization of each individual stack. The dimension of top square mesa is $150 \mu \mathrm{m} \times 150 \mu \mathrm{m}$ and that of bottom one is $200 \mu \mathrm{m} \times 400 \mu \mathrm{m}$. Au/Ge/Ni alloy was evaporated onto the contact area of top mesa, middle and bottom $\mathrm{n}^{+}$layers for ohmic contact formation.

The absorption spectrum and the photocurrent spectra were measured by using a Fourier transform infrared spectrometer. The absolute responsivity was calibrated by using an $800 \mathrm{~K}$ blackbody source. For all photoresponse measurements, the light was coupled through a polished $45^{\circ}$ facet on the substrate of the sample. The temperature of the sample was cooled down with a close-cycled cryostat.

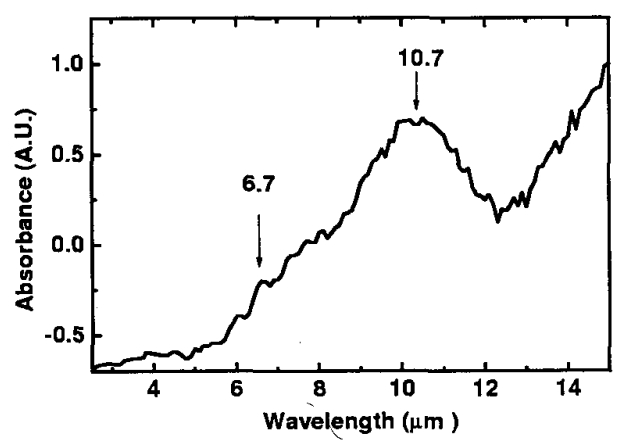

Fig. 2. Room temperature absorption spectrum measured with waveguide geometry of the sample.

\section{Results and discussion}

\section{a. Absorption spectrum}

Figure 2 shows the absorption spectrum of the sample measured at room temperature. Only one peak at 10.7 $\mu \mathrm{m}$ is clearly resolved from the measurement. We identify this peak to be the absorption associated with the intersubband transition at the $\mathrm{BZ}$ zone edge of the bottom SL layer. A less clear peak at $6.7 \mu \mathrm{m}$ should be related to the main peak of the top SL. We should note that for a superlattice structure there are two characteristic peaks, which have been discussed by other authors [10]. The main peak corresponds to the BZ edge transition. Another peak, usually appearing as a shoulder in an absorption spectrum, is related to the zone center transition. However, the zone-center peaks of two SL layers are not resolved from this room temperature measurement.
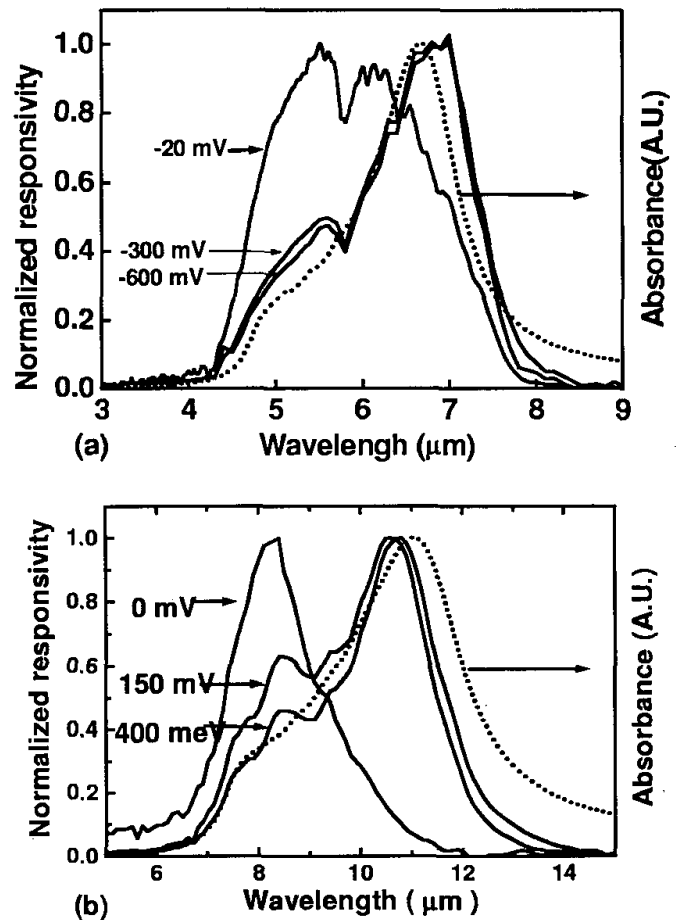

Fig. 3. The normalized response spectra of the top (a) and bottom (b) SLIPs. The dot lines indicated the calculated absorption spectra.

\section{b. Characteristics of individual stacks}

Since our device relies on the tuning and the polarity dependent features in its individual SLIPs, we used the 
middle contact to connect top and bottom SLIP separately to verify their features.

Figure 3 shows the spectral response of each SLIP taken at several bias voltages. We can see that the spectra in both SLIPs, though covering different detection ranges, are tuned in a similar way. At a high bias voltage level, both spectra start to resemble the calculated absorption spectra as shown in Fig 3. The further increase of bias voltage simply increases the magnitude of the responsivity but has little effect on the peak wavelengths and the response lineshapes. The rarely changed lineshapes imply the absorption to be also nearly unchanged under the increasing bias. However, at a low bias voltage level, especially near $0 \mathrm{~V}$, the peak wavelength is at the zone-center peak. We therefore conclude this tuning effect should result form the blocking layer working as an effective voltage-controlled energy filter. The tuning mechanism of the blocking barrier has been discussed in our pervious report [8]. The polarity dependence of the responsivity in the two SLIPs is shown in Fig. 4. Each SLIP is turned on only when the biases have the photocurrent move across its blocking barrier. Due to photovoltaic effect, both SLIPS exhibit a response without external bias.

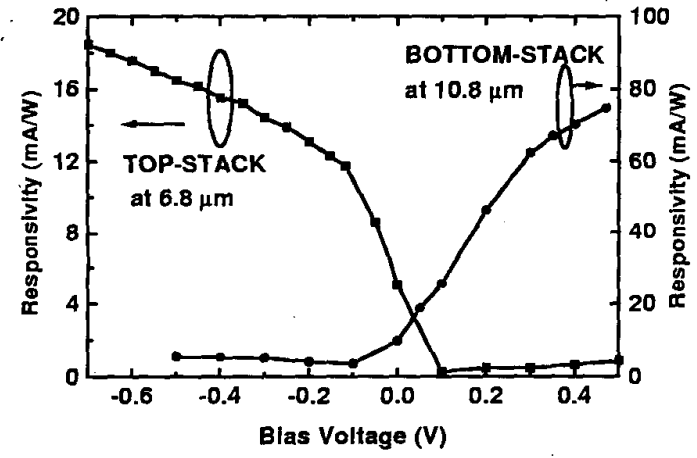

Fig. 4. The voltage-dependence of the responsivity of the individual stacks. Both stacks show the polarity dependence of bias voltage. Each individual stack is tumed on only when the applied voltage has the photoelectrons across the blocking layer.

c. Voltage-controlled photoresponse of the whole detector

Figure 5 shows the normalized response spectra taken at $45 \mathrm{~K}$ under four voltages applied between the top and bottom contacts. Four distinct peaks are resolved. Compared with the spectra of the individual SLIPs in Fig. 3 , we can see that under negative biases the response is contributed from top SLIP while under positives the response comes from the bottom one. The data show the excellent voltage-controlled capability of our device.

Figure 6 shows the voltage dependence of the peak

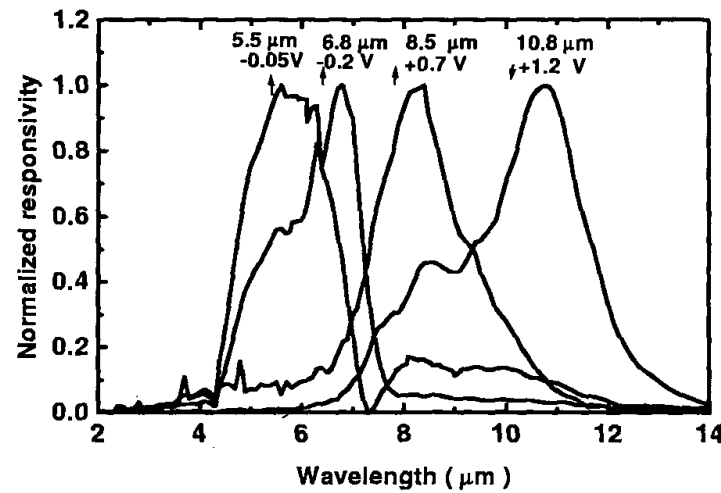

Fig. 5. The spectral response at four bias voltages applied between the top and bottom contacts. The spectra are similar with those spectra measured from individuals shown in Fig. 3.

responsivity at $6.8 \mu \mathrm{m}$ and $10.8 \mu \mathrm{m}$. At the bias range from 0 to $0.6 \mathrm{~V}$, we observe no response from the top stack due to the polarity dependence of the responsivity. At about $0.7 \mathrm{~V}$, the bottom stack starts turning on. At $1.25 \mathrm{~V}$, the peak responsivity at $10.8 \mu \mathrm{m}$ reaches a maximum of about $60 \mathrm{~mA} / \mathrm{W}$ and decreases with further increasing of bias voltage. We can see that the maximum responsivity measured from whole device is less than that measured from the bottom SLIP without stacking. To explain the decreasing in responsivity at higher biases and the reduction of the maximum response, we adopt the equivalent circuit proposed by Liu [6], as drawn in the inset of Fig. 6. According to this model, the voltage dependence of the responsivity of the whole detector can be expressed in terms of the dependence of its component stacks as

$$
R(V, \lambda)=\frac{r_{1} R_{1}\left(V_{1}, \lambda\right)+r_{2} R_{2}\left(V_{2}, \lambda\right)}{r_{1}+r_{2}},
$$

where $\mathbf{R}$ is the responsivity of the whole stacked device, $V$ the applied voltage, $R_{i}$ the responsivity for the $\mathrm{i}_{\text {th }}$ stack, $V_{i}$ the voltage drop across the $\mathrm{i}_{\mathrm{th}}$ stack, $r_{i}$ the differential resistance. Under a given bias voltage $\mathrm{V}$ the voltage $V_{i}$ can be obtained from

$V(I)=V_{1}(I)+V_{2}(I)$

where I is the bias current under the applied voltage V. In Fig. 6, the dot line and solid line represent the responsivity at $10.8 \mu \mathrm{m}$ and $6.8 \mu \mathrm{m}$ estimated from the response of the individual SLIPs according to the equivalent model. The good agreement between the measured response and estimated response justifies the correctness of this model.

In a conventional design, an effective switching from a 


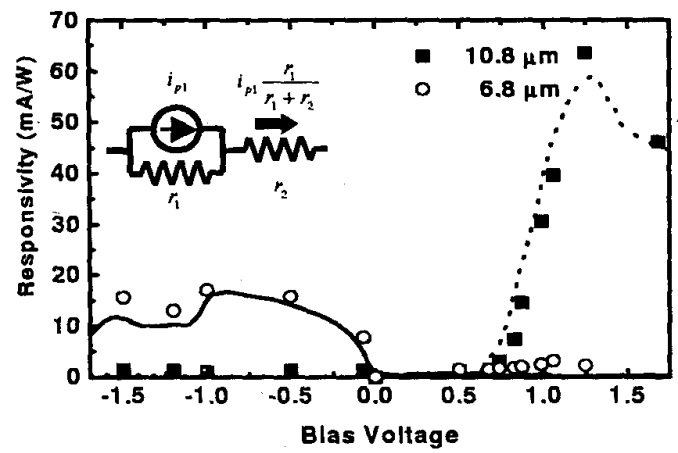

Fig. 6. Measured response at $6.8 \mathrm{um}(0)$ and $10.8 \mathrm{um}(\square)$ plotted versus applied voltage. The solid line and the dot line represent the response estimated from the response of the individual stacks. The estimation is based on the equivalent model as drawn in the inset.

short-wavelength stack to a long-wavelength stack requires the condition of the dynamic resistance of the long-wavelength stack being several times larger than that of the short-wavelength one. The condition is hard to be satisfied in most cases [6,7]. However, in our device the switching can be easily achieved by the contrast of the responsivities due to the opposite turn-on polarity of the bias voltage.

\section{d. Noise performance and detectivity}

The equivalent model above should also work well for the analysis of the noise current. Replacing the photocurrent into the noise current $i_{n}$, we get

$i_{n}^{2}=\frac{r_{1}^{2} i_{n 1}^{2}+r_{2}^{2} i_{n 2}^{2}}{\left(r_{1}+r_{2}\right)^{2}}=2 e I_{D C} \frac{r_{1}^{2}+r_{2}^{2}}{\left(r_{1}+r_{2}\right)^{2}}$,

where $I_{D C}$ is the dc bias current. In the derivation of the above equation, we have used the result of that the noise characteristics in each SLIP is full shot noise [8]. Using the measured responsivity together with the estimated noise current, we estimated the detectivities of the device are $1 \times 10^{9}, 4 \times 10^{9}, 5 \times 10^{9}$ and $1.5 \times 10^{9} \mathrm{~cm}-\mathrm{Hz}^{05} / \mathrm{W}$ at the bias voltages of $-0.05,-0.2,0.7$ and $1.2 \mathrm{~V}$ respectively.
We have investigated the performance of a two-SLIP detector as a voltage-controlled multicolor detector. Using the bias polarity dependence of the photoresponse and the voltage-tunable peak wavelength of each individual SLIP, we successfully select four peak wavelengths at $10.8 \mu \mathrm{m}, 7.6 \mu \mathrm{m}, 6.8 \mu \mathrm{m}$ and $5.4 \mu \mathrm{m}$ by controlling the bias voltage. Compared with the traditional voltage-controlled designs, the detector achieves better switching between response colors and lower operating bias voltage.

This work was supported by the National Science Council of the Republic of China under contract number NSC 88-2112-M-002-030.

\section{Reference}

[1] L. C. West and S. J. Eglash, "First observation of an extremely large-dipole infrared transition within the conduction band of a GaAs quantum well," Appl. Phys. Lett. Vol. 46, pp. 1156-1158, June 1985 .

[2] B.F. Levein, "Quantum -well infrared detectors," J. Appl. Phys. Vol. 74, R1-R81, October 1993

[3] Jung-Chi Chiang, and Sheng S. Li; M. Z. Tidrow; P. Ho, M. Tsai and $C$. P. Lee, "Voltage-tunable triple-coupled InGaAs/GaAs/AIGaAs quantum well infrared photodetector for 8-12 $\mu$ m," Appl. Phys. Lett. Vol. 14, pp. 2412-2415, October 1996.

[4] M. Martinet, F, Luc, E, Rosencher, Ph. Bois, and S. Delaitre, "Electrical tunability of infrared detectors using compositionally asymmetric GaAs/AlGaAs multiquantum wells", Appl. Phys. Lett. Vol. 60, pp. 895-896, February 1992.

[5] I.Grave, A. Shakouri, N. Kuze and A. Yariv, "Voltage-controlled tunable GaAs/AlGaAs multistack quantum well infrared detector," Appl. Phys. Lett. Vol. 60, pp. 2362-2364, May 1992

[6] H.C. Liu, Jianmeng Li, J.R. Thompson, Z.R. Wasilewski, M. Buchanan, and J.G. Simmins, "Multicolor Voltage-tunable Quantum-Well infrared Photodetector," IEEE Electron Devices Lett. Vol. 14, pp. 566-568, December 1993.

[7] K. L. Tsai, C. P. Lee, J.S. Tsang and H.R. Chen, "Two-colour quantum well infrared photodetector with peak responsivity at $3.9 \mu \mathrm{m}$ and $8.1 \mu \mathrm{m}$," Electronics Lett. Vol. 30, pp. 1352-1353, August 1994.

[8] M.C. Hsu, Y.F. Hsu and C. H. Kuan, Proc. SPIE 3269-11, 1999

[9] K. K. Choi, The physics of quantum well infrared photodetector, ch, 3, pp. 93-98.

[10] For example, M. Helm, W.Hilber, T.Fromherz, F.M. Peters, K.Alavi and R.N. Pathak, "Intersubband absoption in strongly coupled superlattices: miniband dispersion, critical points, and oscillator strengths", in Quantum Well Intersubband Transition Physics and Devices, edited by H.C. Liu, B.F. Levine, and J.Y. Anderson, (Kluwer, Netherlands, 1994), pp. 291-300.

Summary 